\title{
Investigating Fontan Failure Using Mathematical Modeling
}

\author{
Matthew Doyle ${ }^{1} \dagger$, Ferran Brosa Planella $^{2}$, Jen Bryson $^{3}$, \\ Brooks Emerick ${ }^{4}$, Daniel Fong ${ }^{5}$, CASEy Johnson ${ }^{6}$, Ayşe Kabatas ${ }^{7}$, \\ Greg Murphy ${ }^{8}$, Tracy Stepien $^{9}$,and Isaac Tate ${ }^{10}$. \\ 1 University Health Network, University of Toronto, Canada \\ ${ }^{2}$ Mathematical Institute, University of Oxford, UK \\ ${ }^{3}$ University of California, Irvine, USA \\ 4 Kutztown University, Pennsylvania, USA \\ 5 US Merchant Marine Academy, USA \\ 6 Claremont Graduate University, California, USA \\ 7 Karadeniz Teknik Universitesi, Turkey \\ 8 Harvey Mudd College, California, USA \\ 9 University of Arizona, USA \\ 10 University of California, Riverside, USA
}

(Communicated to MIIR on 13 August 2021)

Study Group: 34th MPI Workshop, 25-29 June, 2018, Claremont, USA

Communicated by: Hilary Ockendon

Industrial Partner: Toronto General Hospital

Presenter: Matthew Doyle

Team Members: M. Doyle (University of Toronto); F.B. Planella (University of Oxford); J. Bryson (UC Irvine); B. Emerick (Kutztown University); D. Fong (US Merchant Marine Academy); C. Johnson (Claremont Graduate University); A. Kabata (Karadeniz Teknik Universitesi); G. Murphy (Harvey Mudd College); T. Stepien (University of Arizona); I. Tate (UC Riverside)

Industrial Sector: Biomedical/Healthcare

Tools: ODEs, Dynamical systems

Key Words: Fontan circulation

MSC2020 Codes: 34, 92

\section{Summary}

Babies born with a single functioning heart ventricle instead of two require a series of surgeries during the first few years of life to redirect their blood flow, resulting in a Fontan circulation. Patients with Fontan circulations have excellent early survival; however, over time, their circulations begin to fail, ultimately resulting in their death. Currently, the only treatment for failing Fontan circulation is a heart transplant; however, many Fontan patients do not survive long enough to receive a donor heart. One of the reasons for this is a lack of understanding of the Fontan failure cascade. Often patients are identified as failing when they do not have enough time left to receive a heart transplant. The objective of this problem is to develop mathematical models of healthy and failing Fontan circulations to (i) improve our understanding of Fontan failure from a hemodynamic perspective, and (ii) identify physiologically-relevant ranges of parameters.

$\dagger$ Corresponding Author: matthew.gerard.doyle@gmail.com 


\title{
2018 Workshop on Mathematical Problems in Industry Final Report Investigating Fontan Failure using Mathematical Modeling
}

\author{
F. Brosa Planella ${ }^{1}$, J. Bryson ${ }^{2}$, B. Emerick ${ }^{3}$, D. Fong ${ }^{4}$, \\ C. Johnson ${ }^{5}$, A. Kabatas ${ }^{6}$, G. Murphy ${ }^{7}$, T. Stepien ${ }^{8}$, I. Tate ${ }^{9}$ \\ Problem Presenter: M. Doyle ${ }^{10}$
}

December 19, 2018

\section{Problem statement}

Babies born with a single functioning heart ventricle instead of the usual two require a series of surgeries during the first few years of life to redirect their blood flow. The resulting circulation, in which systemic venous blood flows directly into the pulmonary arteries, bypassing the heart, is referred to as the Fontan circulation. Patients with Fontan circulations have excellent early survival; however, over time, their circulations begin to fail, ultimately resulting in their death. Currently, the only treatment for failing Fontan circulation is a heart transplant and due to the relative high risk associated with transplantation in Fontan patients, coupled with the long wait times, many Fontan patients do not survive long enough to receive a donor heart. One of the reasons for this is a lack of understanding of the cascade of Fontan failure. Often patients are only identified as failing when they do not have enough time left to receive a heart transplant. The objective of this problem is to develop mathematical models of healthy and failing Fontan circulations to i) improve our understanding of Fontan failure from a hemodynamic perspective, and ii) identify physiologically-relevant ranges of parameters.

\section{Introduction}

Lumped parameter models are zero-dimensional representations of fluid flow in a system created through analogies between fluid mechanics and electrical circuits. For the Fontan circulation, there have been several previous lumped parameter models $[3,4,5,6]$ which have examined the design of cavopulmonary assist devices [6], the impact of exercise on the Fontan circulation [3], comparisons between two ventricle and single ventricle physiology [4], and changes in hemodynamics following the Fontan procedure [5]. However, to date, none of these models have addressed the cascade of Fontan failure. The long-term goal of the models developed in this study is to generate personalized patient-specific models of the Fontan circulation that can assess the degree of Fontan failure in a given patient and determine potential treatment options at an earlier timepoint than current clinical approaches.

The outline of this report is as follows: in Section 3 we introduce the dimensional and non-dimensional spatially homogeneous ODE model; in Section 4 we analyze an asymptotic approximation of the model;

\footnotetext{
${ }^{1}$ University of Oxford, ${ }^{2}$ University of California, Irvine, ${ }^{3}$ Kutztown University, ${ }^{4}$ US Merchant Marine Academy, ${ }^{5}$ Claremont Graduate University, ${ }^{6}$ Karadeniz Teknik Üniversitesi, ${ }^{7}$ Harvey Mudd College, ${ }^{8}$ University of Arizona, ${ }^{9}$ University of California, Riverside, ${ }^{10}$ University of Toronto
} 


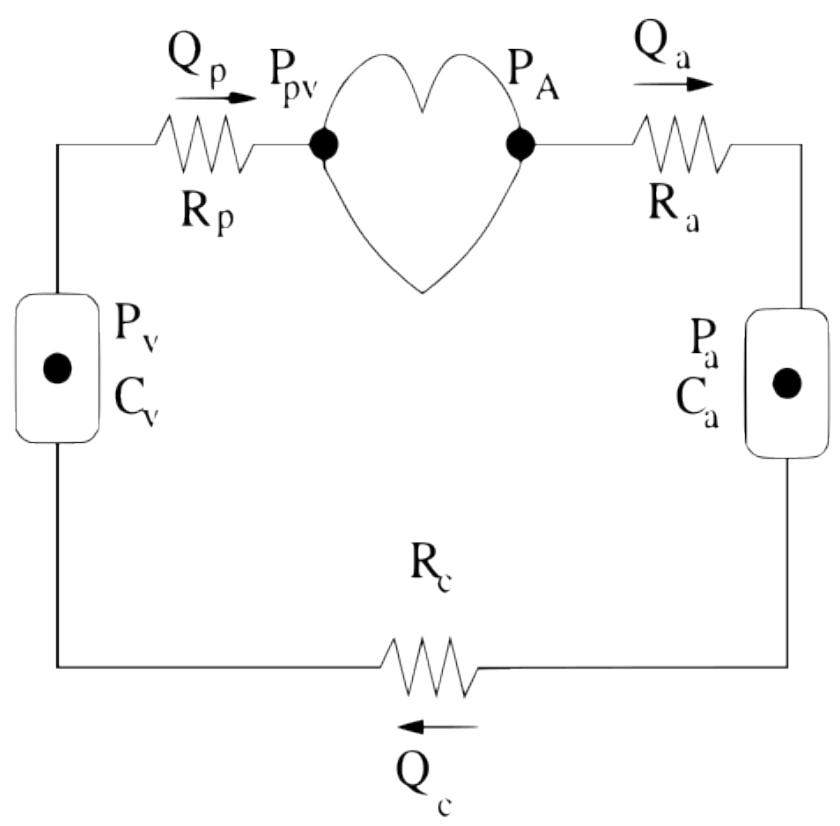

Figure 1: Diagram of the analogous electrical circuit model of Fontan circulation presented in [1]. $R_{j}$ represents the resistances of the blood vessels, $C_{j}$ represents the compliances, $P_{j}$ represents the blood pressures, and $Q_{j}$ represents the blood flows.

in Section 5 we discuss the numerical methods used to solve the dimensional model and show simulation results; in Section 6 we analyze parameter sensitivity of the dimensional model using the LH-OAT method; in Section 7 we extend the dimensional model to include the upper and lower parts of the body and the synthetic extracardiac conduit; and in Section 8 we introduce the spatially inhomogeneous PDE model analogous to the original dimensional model and show simulation results.

\section{Spatially homogeneous ODE model}

We first consider the spatially homogeneous ODE model from [1] for the pressure in a Fontan blood flow circulation. This model is based on the electrical circuit analogy, so it can be represented, as in Figure 1, as a circuit made of resistances and compliances, where the heart is represented as a double compliance that switches periodically in time between two states of diastole and systole and thus inducing a flow in the blood vessels. Diastole is the phase of the heartbeat when the heart muscle relaxes and allows the chambers to fill with blood, and systole is the phase of the heartbeat when the heart muscle contracts and pumps blood from the chambers into the arteries.

In the analogy of the circulatory system to an electrical circuit, the resistances of blood vessels are denoted by parameter $R_{j}$ (which assumes that blood flows through the vessels in response to pressure gradients) and the compliances of blood vessels are denoted by parameter $C_{j}$ (which assumes that blood vessels are elastic and the blood vessel wall is stretched due to pressure differences inside and outside the vessel) [2]. Here, $j=A$ corresponds to the aorta, $j=a$ corresponds to arterial vessels, $j=c$ corresponds to capillaries, $j=v$ corresponds to venous vessels, $j=p$ and $j=p v$ corresponds to the pulmonary vein, $j=d$ corresponds to the heart during diastole, and $j=s$ corresponds to the heart during systole.

The blood pressure is denoted by variable $P_{j}$ and the volume of a blood vessel is denoted by variable $V_{j}$. The blood flow through the blood vessels is denoted by $Q_{j}$. 


\begin{tabular}{|c|c|l|}
\hline Variables & Units & Description \\
\hline$V_{a}$ & $\mathrm{~L}$ & Arterial compliance vessel volume \\
$V_{v}$ & $\mathrm{~L}$ & Venous compliance vessel volume \\
$V_{h}$ & $\mathrm{~L}$ & Heart compliance vessel volume \\
$P_{A}$ & $\mathrm{mmHg}$ & Arterial pressure at heart \\
$P_{a}$ & $\mathrm{mmHg}$ & Arterial pressure \\
$P_{v}$ & $\mathrm{mmHg}$ & Venous pressure \\
$P_{p v}$ & $\mathrm{mmHg}$ & Pulmonary pressure at heart \\
\hline
\end{tabular}

Table 1: Table of the variables in the ODE model (1)-(2).

\subsection{Dimensional model}

From [1], the equations for the pressure during diastole, where $0<t<t_{1}$ with $t_{1}$ being the time spent in diastole, are

$$
\begin{aligned}
C_{a} \frac{\mathrm{d} P_{a}}{\mathrm{~d} t} & =-\frac{P_{a}-P_{v}}{R_{c}}, \\
C_{v} \frac{\mathrm{d} P_{v}}{\mathrm{~d} t} & =\frac{P_{a}-P_{c}}{R_{c}}-\frac{P_{v}-P_{p v}}{R_{p}}, \\
P_{p v} & =\frac{1}{C_{d}}\left(V_{T}-V_{a}^{0}-C_{a} P_{a}-V_{v}^{0}-C_{v} P_{v}-V_{d}^{0}\right), \\
P_{A} & =P_{a},
\end{aligned}
$$

and during systole, where $t_{1}<t<t_{2}$ with $t_{2}-t_{1}$ being the time spent in systole, are

$$
\begin{aligned}
C_{a} \frac{\mathrm{d} P_{a}}{\mathrm{~d} t} & =\frac{P_{A}-P_{a}}{R_{a}}-\frac{P_{a}-P_{c}}{R_{c}}, \\
C_{v} \frac{\mathrm{d} P_{v}}{\mathrm{~d} t} & =\frac{P_{a}-P_{c}}{R_{c}}, \\
P_{A} & =\frac{1}{C_{s}}\left(V_{T}-V_{a}^{0}-C_{a} P_{a}-V_{v}^{0}-C_{v} P_{v}-V_{s}^{0}\right), \\
P_{p v} & =P_{v},
\end{aligned}
$$

where the variables are defined in Table 1 and the parameters are defined and typical values given in Table 2 . The initial conditions are $P_{a}(0)=P_{a 0}$ and $P_{v}(0)=P_{v 0}$.

In addition to systole and diastole, the heart cycle has two additional phases, isovolumetric contraction and isovolumetric relaxation, during which both heart valves are closed, the blood volume in the heart is constant, and the pressure changes in response to changes in the heart's shape. More specifically, isovolumetric contraction occurs between the end of diastole and the start of systole, during which the heart muscles contract leading to an increase in ventricular pressure. Isovolumetric relaxation occurs between the end of systole and the start of diastole, during which the heart muscles relax leading to a decrease in ventricular pressure. The volume in the heart is given by

$$
V_{h}=V_{T}-\left(V_{a}^{0}+V_{v}^{0}+C_{a} P_{a}+C_{v} P_{v}\right),
$$

and the pressure is given by

$$
P_{h}= \begin{cases}\frac{V_{h}-V_{d}^{0}}{C_{d}}, & \text { during diastole } \\ \frac{V_{h}-V_{s}^{0}}{C_{s}}, & \text { during systole. }\end{cases}
$$




\begin{tabular}{|c|c|c|l|}
\hline Parameters & Typical value & Units & Description \\
\hline$V_{a}^{0}$ & 1.6 & $\mathrm{~L}$ & Basal arterial volume \\
$V_{v}^{0}$ & 3.2 & $\mathrm{~L}$ & Basal venous compliance vessel volume \\
$V_{d}^{0}$ & 0 & $\mathrm{~L}$ & Basal heart volume in diastolic phase \\
$V_{s}^{0}$ & -0.5 & $\mathrm{~L}$ & Basal heart volume in systolic phase \\
$V_{T}$ & 6.3325 & $\mathrm{~L}$ & Total volume of the vessel system \\
$C_{a}$ & 0.00125 & $\mathrm{~L} \mathrm{mmHg}^{-1}$ & Arterial compliance \\
$C_{v}$ & 0.0625 & $\mathrm{~L} \mathrm{mmHg}^{-1}$ & Venous compliance \\
$C_{d}$ & 0.01 & $\mathrm{~L} \mathrm{mmHg}^{-1}$ & Heart compliance during diastole \\
$C_{s}$ & 0.005 & $\mathrm{~L} \mathrm{mmHg}^{-1}$ & Heart compliance during systole \\
$R_{a}$ & 0.5 & $\mathrm{mmHg} \mathrm{min} \mathrm{L}^{-1}$ & Arterial resistance \\
$R_{p}$ & 2.9 & $\mathrm{mmHg} \mathrm{min} \mathrm{L}^{-1}$ & Pulmonary resistance \\
$R_{c}$ & 17 & $\mathrm{mmHg} \mathrm{min} \mathrm{L}^{-1}$ & Capillary resistance \\
\hline
\end{tabular}

Table 2: Table of the typical parameter values of the ODE model (1)-(2).

\section{$3.2 \quad$ Non-dimensional model}

To mathematically analyze the dimensional model (1)-(2), we proceed to non-dimensionalise it to reduce the number of parameters and identify the importance of individual terms. We take the following scalings for pressure and time

$$
P_{j}=\Delta P \hat{P}_{j} \quad \text { and } \quad t=\Delta t \hat{t}, \quad j=a, v, A, p v,
$$

where $\Delta P$ and $\Delta t$ are the characteristic scalings of pressure and time. We choose $\Delta P \approx 120 \mathrm{mmHg}$ which is a typical value for $P_{a}$ and $\Delta t=t_{d}+t_{s} \approx 60^{-1}$ min which is the period of a heartbeat.

Substituting the scalings (5) into the dimensional model (1)-(2) and dropping hats, we obtain the dimensionless model for the Fontan circulation. During diastole $\left(0<t<t^{*}\right)$ we have

$$
\begin{aligned}
\mu_{a} \frac{\mathrm{d} P_{a}}{\mathrm{~d} t} & =-\left(P_{a}-P_{v}\right) \\
\mu_{v} \frac{\mathrm{d} P_{v}}{\mathrm{~d} t} & =\left(P_{a}-P_{v}\right)-\rho_{p}\left(P_{v}-P_{p v}\right) \\
P_{p v} & =\varphi_{T}-\varphi_{a}^{0}-\varphi_{v}^{0}-\varphi_{d}^{0}-\gamma_{a} P_{a}-\gamma_{v} P_{v} \\
P_{A} & =P_{a}
\end{aligned}
$$

and during systole $\left(t^{*}<t<1\right)$ we have

$$
\begin{aligned}
\mu_{a} \frac{\mathrm{d} P_{a}}{\mathrm{~d} t} & =\rho_{a}\left(P_{A}-P_{a}\right)-\left(P_{a}-P_{v}\right), \\
\mu_{v} \frac{\mathrm{d} P_{v}}{\mathrm{~d} t} & =P_{a}-P_{v}, \\
P_{A} & =\gamma_{s}^{-1}\left(\varphi_{T}-\varphi_{a}^{0}-\varphi_{v}^{0}-\varphi_{s}^{0}-\gamma_{a} P_{a}-\gamma_{v} P_{v}\right), \\
P_{p v} & =P_{v},
\end{aligned}
$$


where we have defined the dimensionless parameters

$$
\begin{array}{rlll}
\mu_{a} & =\frac{C_{a} R_{c}}{\Delta t} \approx 1.28, \quad \mu_{v}=\frac{C_{v} R_{c}}{\Delta t} \approx 63.75, & \rho_{p}=\frac{R_{c}}{R_{p}} \approx 5.86, & \rho_{a}=\frac{R_{c}}{R_{a}} \approx 34, \\
\varphi_{a}^{0}=\frac{V_{a}^{0}}{\Delta P C_{d}} \approx 1.33, & \varphi_{v}^{0}=\frac{V_{v}^{0}}{\Delta P C_{d}} \approx 2.67, & \varphi_{s}^{0}=\frac{V_{s}^{0}}{\Delta P C_{d}} \approx-0.42, & \varphi_{d}^{0}=\frac{V_{d}^{0}}{\Delta P C_{d}} \approx 0, \\
\varphi_{T}=\frac{V_{T}}{\Delta P C_{d}} \approx 5.28, & \gamma_{a}=\frac{C_{a}}{C_{d}} \approx 0.13, & \gamma_{v}=\frac{C_{v}}{C_{d}} \approx 6.25, & \gamma_{s}=\frac{C_{s}}{C_{d}} \approx 0.5,
\end{array}
$$

and the approximate values have been calculated using the parameter values given in Table 2 and the scalings in (5). The initial conditions are $P_{a}(0)=P_{a 0}$ and $P_{v}(0)=P_{v 0}$, where now $P_{a 0}$ and $P_{v 0}$ are dimensionless pressures.

The dimensionless equations for the isovolumetric phases of the heart are the following. The volume is given by

$$
V_{h}=\phi_{T}-\left(\phi_{a}^{0}+\phi_{v}^{0}+\gamma_{a} P_{a}+\gamma_{v} P_{v}\right),
$$

and the pressure is

$$
P_{h}= \begin{cases}V_{h}-\varphi_{d}^{0}, & \text { during diastole } \\ \gamma_{s}^{-1}\left(V_{h}-\varphi_{s}^{0}\right), & \text { during systole }\end{cases}
$$

Notice that the value of $\mu_{v}$ is significantly large, so this is the parameter we use for the asymptotic approximation of the model. One could take $\rho_{a}$ as a large parameter too, but this leads to the introduction of transition layers, which adds complexity to the analysis rather than simplifying it, so we do not take the limit $\rho_{a} \rightarrow+\infty$ here.

\section{Reduced model for large $\mu_{v}$}

Even though expressions for the exact analytical solutions to the model can be found, their expressions are very convoluted and therefore they are not very enlightening. In this section we study the reduced model for large $\mu_{v}$, which physically means that the time scale of the RC circuit formed by the resistance $R_{c}$ and the compliance $C_{v}$ is much larger than the time scale of a heart beat. Because the response of the venous compliance is very slow, the pressure in the veins $P_{v}$ can be taken to be constant, and the system reduces to a single ODE for $P_{a}$.

Taking the limit $\mu_{v} \rightarrow+\infty$, the model reduces to the following. During diastole $\left(0<t<t^{*}\right)$ we have

$$
\begin{aligned}
\mu_{a} \frac{\mathrm{d} P_{a}}{\mathrm{~d} t} & =-\left(P_{a}-P_{v}\right), \\
\frac{\mathrm{d} P_{v}}{\mathrm{~d} t} & =0 \\
P_{p v} & =\varphi_{T}-\varphi_{a}^{0}-\varphi_{v}^{0}-\varphi_{d}^{0}-\gamma_{a} P_{a}-\gamma_{v} P_{v} \\
P_{A} & =P_{a}
\end{aligned}
$$

and during systole $\left(t^{*}<t<1\right)$ we have

$$
\begin{aligned}
\mu_{a} \frac{\mathrm{d} P_{a}}{\mathrm{~d} t} & =\rho_{a}\left(P_{A}-P_{a}\right)-\left(P_{a}-P_{v}\right), \\
\frac{\mathrm{d} P_{v}}{\mathrm{~d} t} & =0, \\
P_{A} & =\gamma_{s}^{-1}\left(\varphi_{T}-\varphi_{a}^{0}-\varphi_{v}^{0}-\varphi_{s}^{0}-\gamma_{a} P_{a}-\gamma_{v} P_{v}\right), \\
P_{p v} & =P_{v},
\end{aligned}
$$

with initial conditions $P_{a}(0)=P_{a 0}$ and $P_{v}(0)=P_{v 0}$. 
Now we can find simple analytical solutions to the system which are given by the following expressions. During diastole $\left(0<t<t^{*}\right)$ we have

$$
\begin{aligned}
P_{a} & =P_{v 0}+\left(P_{a 0}-P_{v 0}\right) e^{-\frac{t}{\mu_{a}}} \\
P_{v} & =P_{v 0} \\
P_{p v} & =\varphi_{T}-\varphi_{a}^{0}-\varphi_{v}^{0}-\varphi_{d}^{0}-\gamma_{v} P_{v 0}-\gamma_{a} P_{a} \\
P_{A} & =P_{a}
\end{aligned}
$$

and during systole $\left(t^{*}<t<1\right)$ we have

$$
\begin{aligned}
P_{a} & =\frac{P_{v 0}+A \rho_{a}}{1+\rho_{a}+B \rho_{a}}+\left(P_{v 0}+\left(P_{a 0}-P_{v 0}\right) e^{-\frac{t^{*}}{\mu_{a}}}-\frac{P_{v 0}+A \rho_{a}}{1+\rho_{a}+B \rho_{a}}\right) e^{-\frac{1+\rho_{a}+B \rho_{a}}{\mu_{a}}\left(t-t^{*}\right)}, \\
P_{v} & =P_{v 0} \\
P_{A} & =A-B P_{a}, \\
P_{p v} & =P_{v 0},
\end{aligned}
$$

where

$$
\begin{aligned}
& A=\gamma_{s}^{-1}\left(\varphi_{T}-\varphi_{a}^{0}-\varphi_{v}^{0}-\varphi_{s}^{0}-\gamma_{v} P_{v 0}\right), \\
& B=\frac{\gamma_{a}}{\gamma_{s}}
\end{aligned}
$$

The first thing we notice is that $P_{v}$ is constant throughout the whole cycle. This is due to the disparity of time scales (which is reflected in the fact that $\mu_{v}$ is large), which means that we can take the quasisteady state assumption for $P_{v}$. We notice as well that the system is mainly driven by $P_{a}$ and thus the key parameters of the system are those related to the arterial system.

\subsection{Comparison with numerical simulations}

Now we can compare the re-dimensionalised analytic solutions (13)-(14) with the numerical simulations of the full problem (1)-(2). In Figure 2 we show the analytic profile pressures given by the analytic solutions (13)(14) during one heartbeat. In Figure 3 we show the comparison between analytic solutions and numerical simulations of (1)-(2). We observe quite good agreement for all the pressures (take note of the vertical scales). The analytic approximations can be improved by taking the next order term in the asymptotic expansion.

\subsection{Poincaré maps for $P_{a}$}

In the previous sections we determined solutions for a single heartbeat. However, we are interested in patching several of these solutions together to reproduce the evolution in time of the pressure over several cycles and how it converges to the limit cycle. In order to do so, we need to prescribe the pressure at the beginning of each diastole cycle. This motivates the derivation of the Poincaré maps for the pressure $P_{a}$ presented in this section. These maps take the pressure at a certain time $t_{0}$ and map it to the pressure at the same time in the next heartbeat $t_{0}+\Delta t$. In particular we want to study the maps at the beginning of diastole and systole. In particular, the Poincaré maps are useful to understand the convergence to a limit cycle and to patch several instances of the analytical solutions to produce pressure profiles over several heartbeats.

The Poincaré map $f$ is formalised as

$$
f\left(P\left(t_{n}\right)\right)=P\left(t_{n}+\Delta t\right),
$$

where $t_{n}$ is a certain time and $\Delta t$ is the period of a heart beat. We can define $t_{n+1}=t_{n}+\Delta t$ and $P^{n}=P\left(t_{n}\right)$. Then, we can define the Poincaré map using the notation

$$
P^{n+1}=f\left(P^{n}\right)
$$




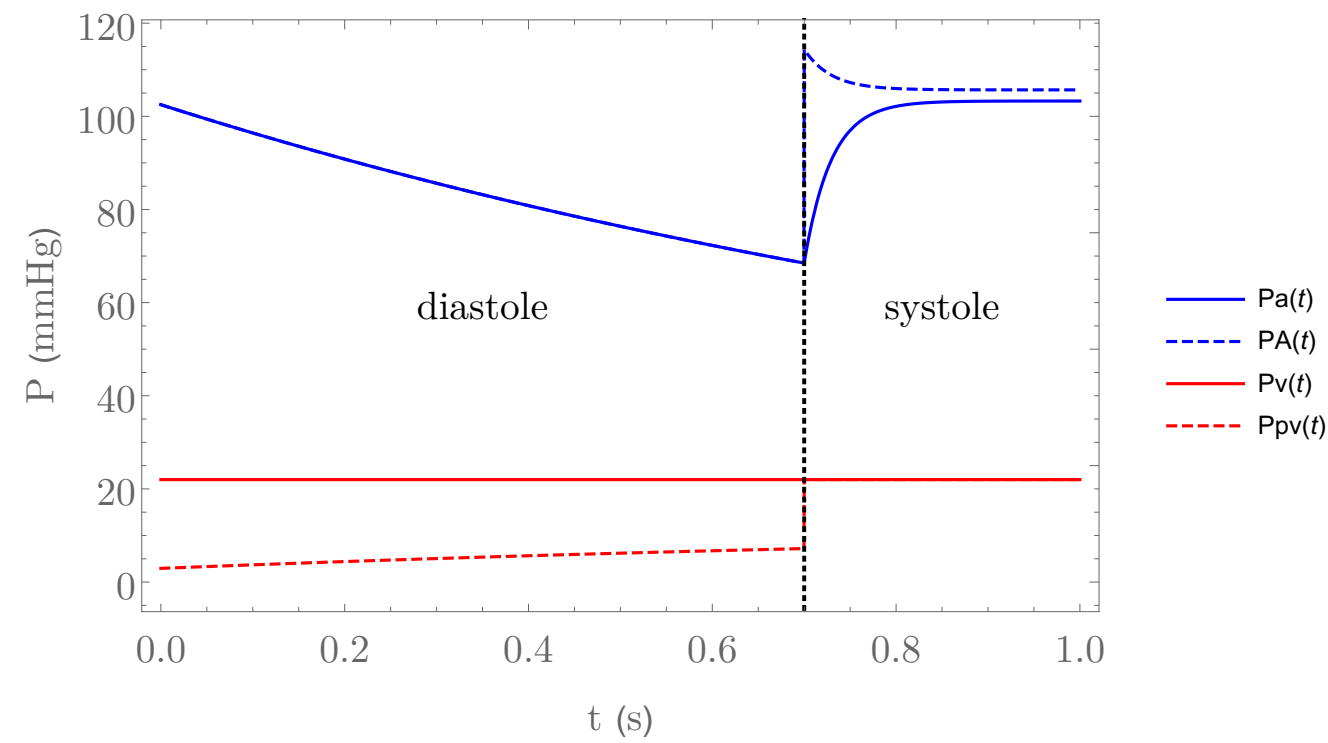

Figure 2: Pressure profiles given by the asymptotic solutions (13)-(14) during one heartbeat. As prescribed by the solutions, during diastole $P_{A}=P_{a}$ (see (13)) and during systole $P_{p v}=P_{v}$ (see (14)).

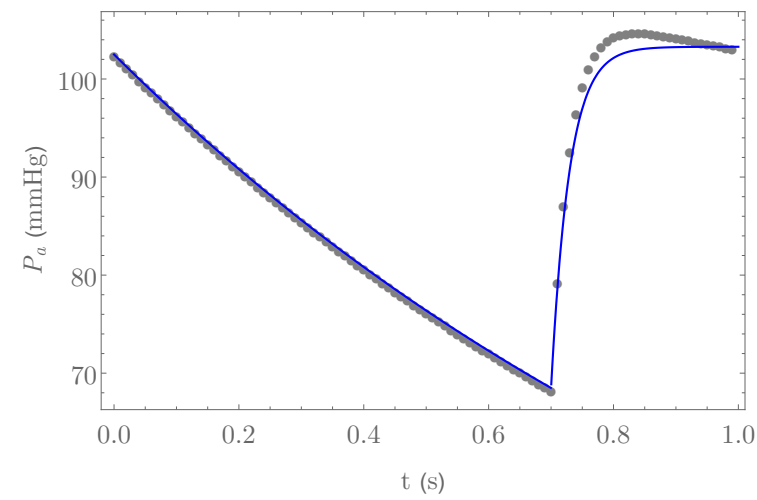

(a) $P_{a}(t)$

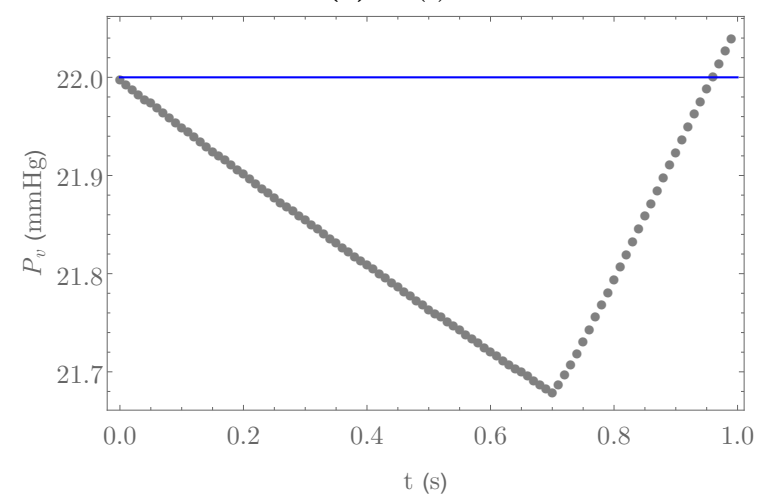

(c) $P_{v}(t)$

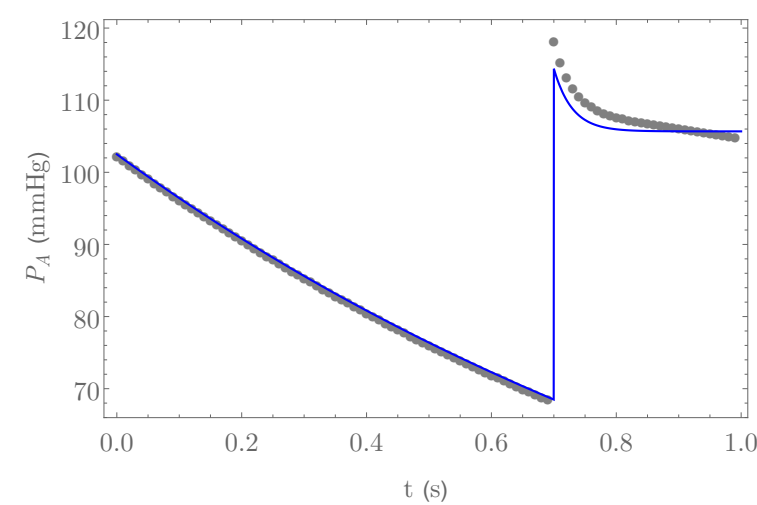

(b) $P_{A}(t)$

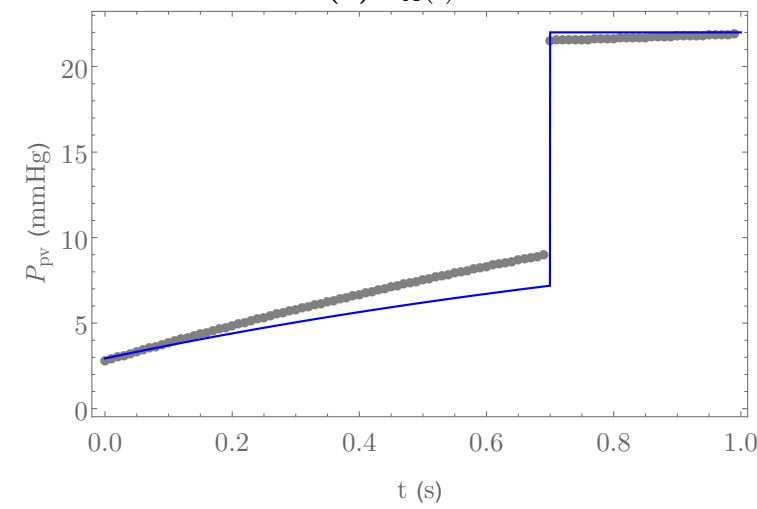

(d) $P_{p v}(t)$

\section{— asymptotics $\bullet$ numerics}

Figure 3: Comparison between asymptotic solutions (13)-(14) and numerical simulations of (1)-(2) for each of the four pressures of the model, $P_{a}, P_{A}, P_{v}$, and $P_{p v}$. 
The map for the pressures at the beginning of diastole can be immediately found from (14a) if we consider the pressure at $t=1$ as a function of the initial pressure $P_{a 0}$. We find

$$
P^{n+1}=\frac{P_{v 0}+A \rho_{a}}{1+\rho_{a}+B \rho_{a}}+\left(P_{v 0}+\left(P^{n}-P_{v 0}\right) e^{-\frac{t^{*}}{\mu_{a}}}-\frac{P_{v 0}+A \rho_{a}}{1+\rho_{a}+B \rho_{a}}\right) e^{-\frac{1+\rho_{a}+B \rho_{a}}{\mu_{a}}\left(1-t^{*}\right)} .
$$

We can proceed similarly for the pressure at the beginning of systole. In order to do so, we take $P_{n}$ to be the pressure at the beginning of systole and then patch the solution for systolic pressure with the solution for diastolic pressure. The pressure at the end of the cycle $P^{n+1}$ (which, again, is the pressure at the beginning of systole) as a function of $P^{n}$ provides the Poincare map for the pressure at the beginning of systole, which is

$$
P^{n+1}=P_{v 0}+\left(\frac{P_{v 0}+A \rho_{a}}{1+\rho_{a}+B \rho_{a}}+\left(P^{n}-\frac{P_{v 0}+A \rho_{a}}{1+\rho_{a}+B \rho_{a}}\right) e^{-\frac{1+\rho_{a}+B \rho_{a}}{\mu_{a}}\left(1-t^{*}\right)}-P_{v 0}\right) e^{-\frac{t^{*}}{\mu_{a}}} .
$$

Notice that both maps are linear, so we can rearrange them into

$$
\begin{aligned}
& f_{\text {dia }}(P)=a P+b_{\text {dia }}, \\
& f_{\text {sys }}(P)=a P+b_{\text {sys }},
\end{aligned}
$$

where

$$
\begin{aligned}
a & =e^{-\frac{1}{\mu_{a}}\left(t^{*}+\left(1+\rho_{a}+B \rho_{a}\right)\left(1-t^{*}\right)\right)}, \\
b_{d i a} & =\frac{P_{v 0}+A \rho_{a}}{1+\rho_{a}+B \rho_{a}}+\left(\left(1-e^{-\frac{t^{*}}{\mu_{a}}}\right) P_{v 0}-\frac{P_{v 0}+A \rho_{a}}{1+\rho_{a}+B \rho_{a}}\right) e^{-\frac{1+\rho_{a}+B \rho_{a}}{\mu_{a}}\left(1-t^{*}\right)}, \\
b_{\text {sys }} & =P_{v 0}+\left(\left(1-e^{-\frac{1+\rho_{a}+B \rho_{a}}{\mu_{a}}\left(1-t^{*}\right)}\right) \frac{P_{v 0}+A \rho_{a}}{1+\rho_{a}+B \rho_{a}}-P_{v 0}\right) e^{-\frac{t^{*}}{\mu_{a}}} .
\end{aligned}
$$

For any (positive) parameter values, $a<1$ and therefore the maps have a fixed point and thus the system reaches a limit cycle. Moreover, we can determine the value of those fixed points which is given by

$$
P_{d i a}^{*}=\frac{b_{d i a}}{1-a} \quad \text { and } \quad P_{s y s}^{*}=\frac{b_{\text {sys }}}{1-a} .
$$

For the parameter values in Table 2 we find the values

$$
a=2.07 \times 10^{-5}, \quad b_{d i a}=0.86 \quad \text { and } \quad b_{\text {sys }}=0.57,
$$

which give the steady states

$$
P_{d i a}^{*}=0.86 \quad \text { and } \quad P_{s y s}^{*}=0.57 .
$$

Because $a$ is very small, from (22) we can conclude that the system converges very quickly to the steady state, which is very close to the corresponding value of $b_{j}$. The analysis performed here could be applied as well to the full system (1)-(2), giving analogous results, but the results would be more convoluted because in that case we have a two-dimensional system of ODEs for $P_{a}$ and $P_{v}$.

\section{$5 \quad$ Numerical simulations of dimensional model}

Besides analytically exploring analytic solutions of the model (1)-(2), we also numerically solved the model to obtain the pressure profiles. We solved this system of equations numerically in MATLAB in the m-file denoted by TGH_ODE_Model (see Table 3 for a brief description of MATLAB programs we developed). This file effectively solves the ODE system (1)-(2) by defining piecewise function handles for $V_{h}, P_{p v}$, and $P_{A}$. We used the stiff ODE solver ode23s to solve the system on both the diastolic and systolic time phases. This 


\begin{tabular}{|c|c|}
\hline Program Name & Description \\
\hline TGH_ODE_Model & Simplified ODE solver with figure output \\
\hline TGH_ODE_Function & $\begin{array}{l}\text { Simplified ODE solving function with } \\
\text { solution output }\end{array}$ \\
\hline Pairwise_Parameter_Sensitivity & $\begin{array}{l}\text { Pairwise parameter sweep with average } \\
\text { value and viable parameter space output }\end{array}$ \\
\hline
\end{tabular}

Table 3: Brief description of MATLAB programs.

m-file outputs the solutions $P_{a}$ and $P_{v}$ for a single set of parameter values. It also defines the heart pressure $P_{h}$ as

$$
P_{h}= \begin{cases}\frac{1}{C_{d}}\left(V_{h}-V_{d}^{0}\right), & \text { during diastole } \\ \frac{1}{C_{s}}\left(V_{h}-V_{s}^{0}\right), & \text { during systole. }\end{cases}
$$

This file outputs a variety of graphs that pertain to the pressures, volumes, and cardiac output (i.e. flows) from the system over a span of $\mathrm{N}$ minutes.

We also created a MATLAB function named TGH_ODE_Function that utilizes TGH_ODE_Model but in function form. The inputs of the function are the vector of parameters $\mathrm{x}$ and the duration, measured in minutes, for which the model simulates, denoted as $\mathrm{N}$. The vector of input parameters is defined as the following:

$$
\mathrm{x}=[\mathrm{VaO} \operatorname{Vv0} \mathrm{VdO} \operatorname{Vs} 0 \mathrm{Ra} \operatorname{Rp} \mathrm{Rc} \mathrm{Ca} \mathrm{Cv} \mathrm{Cd} \mathrm{Cs} \mathrm{VT}] ;
$$

The output of this function is a set of solutions for all volumes, including $P_{h}$, the heart volume $\left(V_{h}\right)$, and the flows. The input and output of the function have the following form:

$$
\begin{array}{ll}
\text { INPUT: } & (\mathrm{x}, \mathrm{N}) \\
\text { OUTPUT: } & {[\mathrm{Pa}, \mathrm{Pv}, \mathrm{PA}, \mathrm{Ppv}, \mathrm{Ph}, \mathrm{Vh}, \mathrm{Qa}, \mathrm{Qc}, \mathrm{Qp}]}
\end{array}
$$

Finally, to determine in which regions of parameter space the model output is deemed "healthy" or "failing" Fontan circulation we created a parameter-sweeping m-file defined as Pairwise_Parameter_Sensitivity. This file defines two vectors of parameter values where one parameter is varied and the other parameters are held constant at the baseline values given in Table 2. The file then loops through the two parameter vectors and utilizes TGH_ODE_Function to obtain a solution to the ODE system for each parameters set. To help us understand how varying the parameters results in healthy or failing Fontan circulation, we stored several quantities including the average cardiac output $\left(\bar{Q}_{c}\right)$ and the average arterial pressure $\left(\bar{P}_{a}\right)$.

\section{$5.1 \quad$ Results}

Our main numerical results include a robust ODE solver implementation in MATLAB and a pairwise parameter variation that creates regions of parameter space that map out healthy and failing Fontan circulation based on the model (1)-(2). A typical output of TGH_ODE_Model yields a time series plot of the heart pressures, the cardiac pressure-volume cycle, the heart pressure cycle, and the capillary flow. Figure 4 shows the output that compares well to the results in [1].

Our results of the pairwise parameter variations are depicted in Figures 5-6. To obtain these figures, we looped through two parameter vectors and used the output of the ODE solving program to characterize a healthy or failing Fontan output. A typical output of our model yields a surface, whose height is either the mean arterial pressure, $\bar{P}_{a}$, or the mean capillary output, $\bar{Q}_{c}$. We typically varied a set of two parameters over a log scale, which may span from $10^{-3}$ to $10^{3}$. Contour boundaries for each output are depicted with the solid and dotted black lines. The regions in green are the regions of parameter space for which the parameters yield a healthy, or physiologically sound, Fontan circulation. 

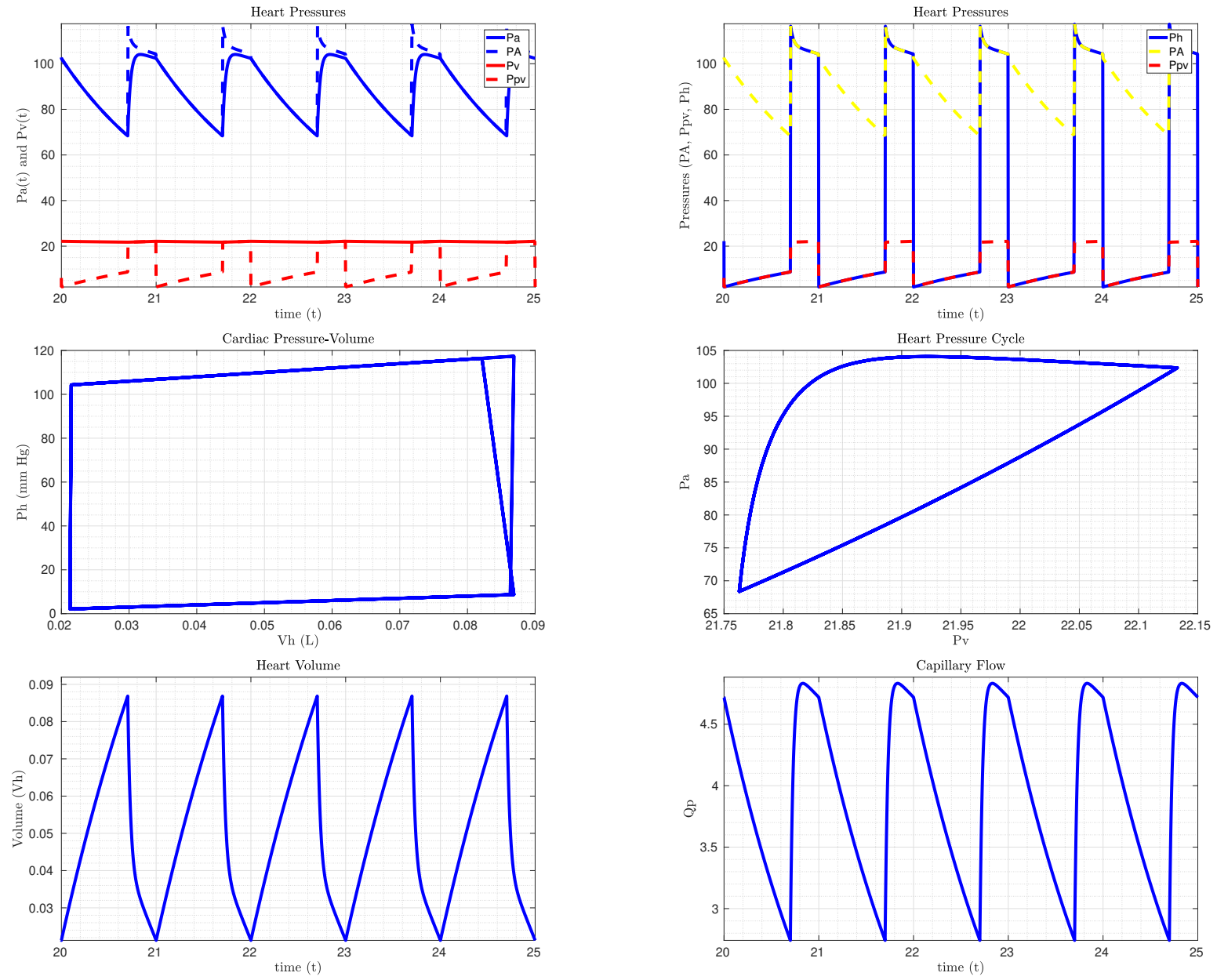

Figure 4: Typical output for a single run of the ODE model (1)-(2). Parameter values are as stated in Table 2. 

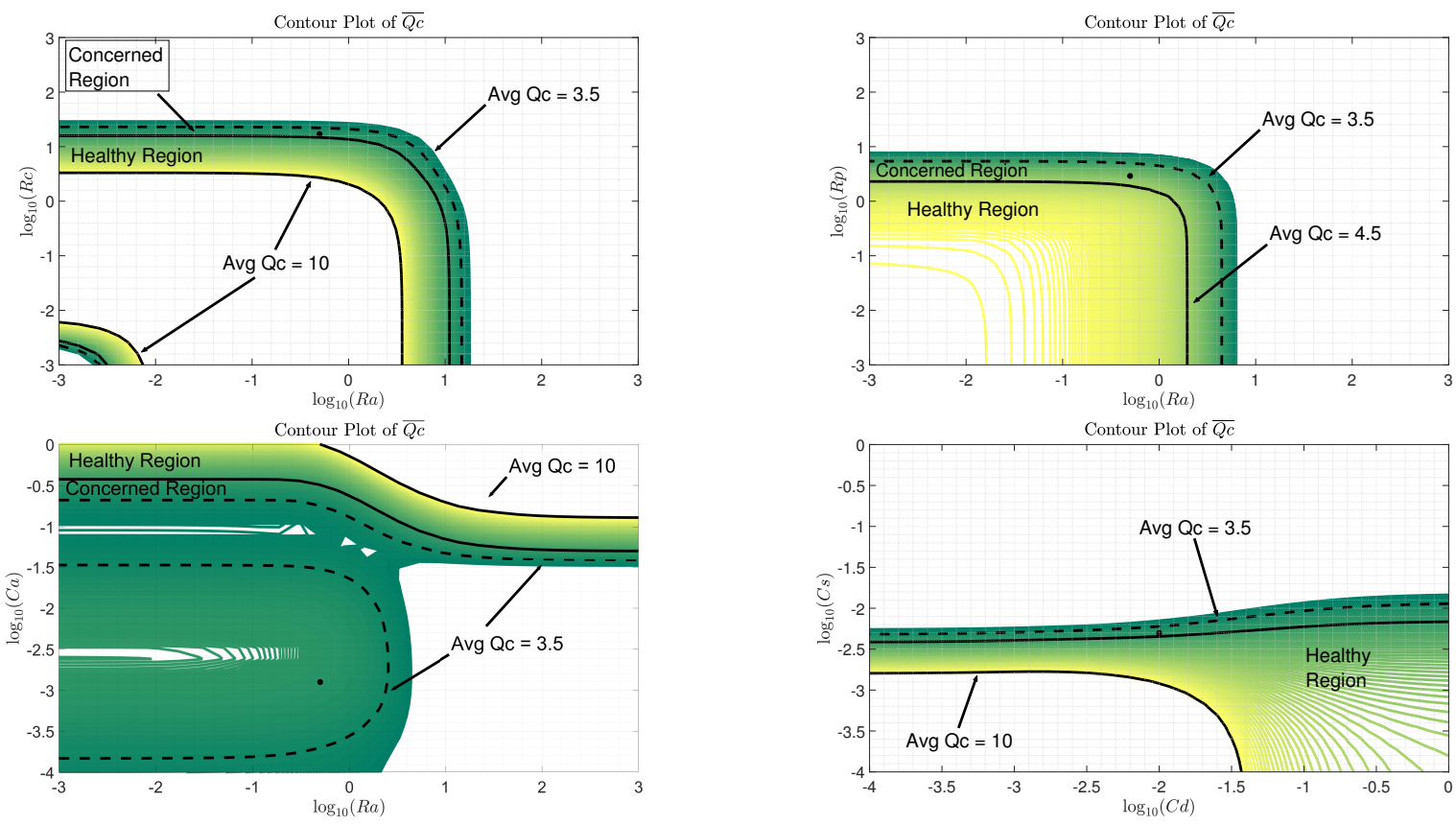

Figure 5: Contour plots of mean capillary flow, $\bar{Q}_{c}$, with variation in model parameters on a log scale.
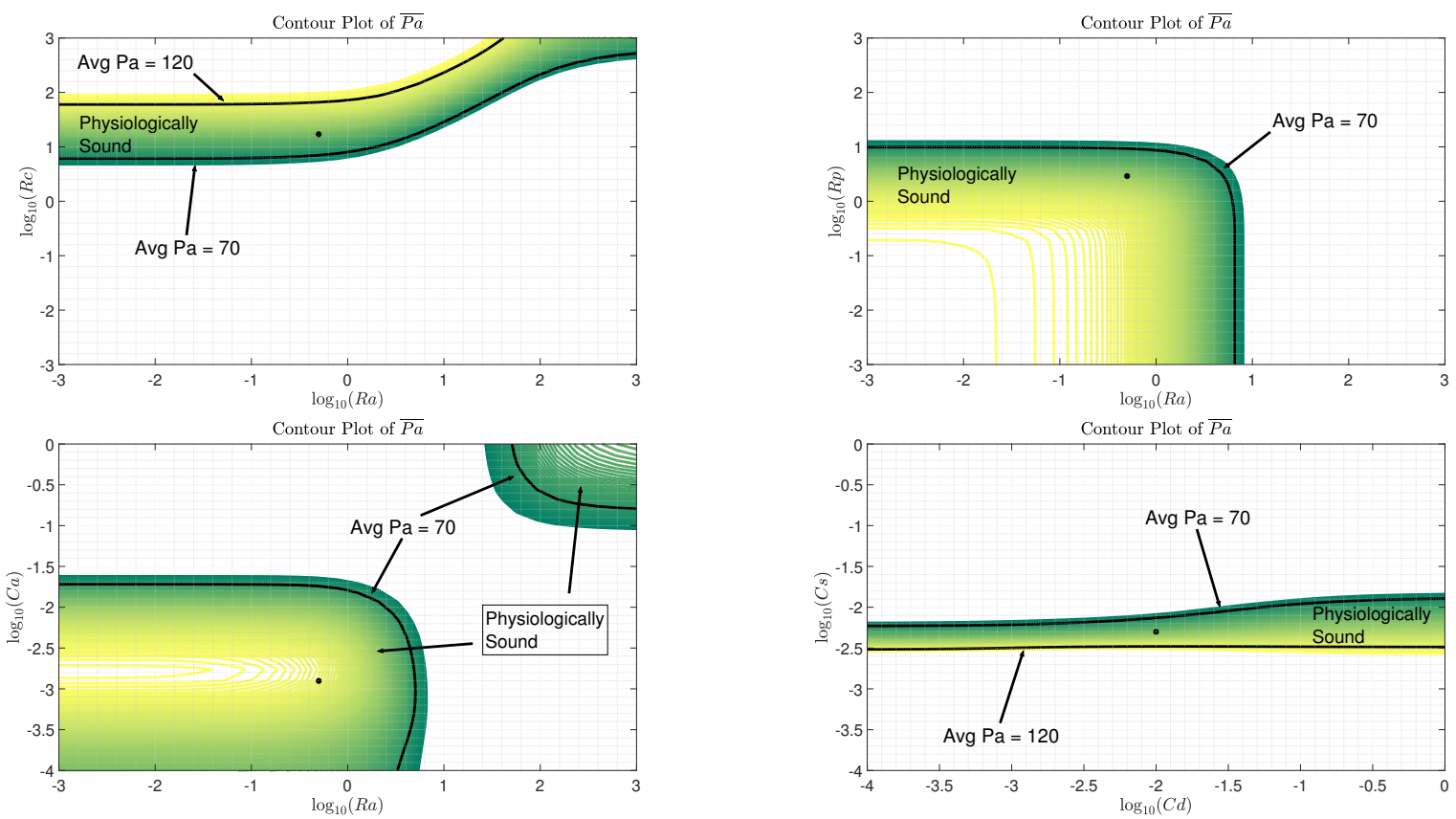

Figure 6: Contour plots of mean arterial pressure, $\bar{P}_{a}$, with variation in model parameters on a log scale. 


\section{Parameter sensitivity of the dimensional model using the LH- OAT method}

To study the behavior of the model (1)-(2), we also analyzed parameter sensitivity to determine which parameters have the most impact on the resulting model output. In particular, we implement the LH-OAT method where "LH" stands for Latin Hypercube and "OAT" stands for One-factor-ㅅt-a-Time [4, 5, 7].

This method first performs LH sampling, which involves taking $N$ Latin hypercube sample points for $N$ intervals. Latin hypercube sampling is a method for generating a near-random sample of parameter values from a multidimensional distribution, which allows for us to sample the multi-dimensional parameter space for our model efficiently.

The method then performs OAT sampling, which involves taking each LH point and varying it $P$ times by changing each of the $P$ parameters one at a time. For each LH point, a local sensitivity analysis is performed by quantifying the changes in model outputs (variables) with respect to changes in model parameters. In particular, for an LH point $\mathbf{X}_{0}=\left(x_{1}, \ldots, x_{P}\right)$, the sensitivity index $S_{i j}$ for parameter $i$ and model output (variable) $j$ is defined as

$$
S_{i j}=\left.\frac{\partial M_{j}(\mathbf{X})}{\partial x_{i}}\right|_{\mathbf{X}=\mathbf{X}_{0}},
$$

where $M$ represents the value of a model output (variable). Because the LH points are discrete, we discretize the partial derivative. One such option is

$$
S_{i j} \approx \frac{M_{j}\left(x_{1}, \ldots, x_{i}+\Delta x_{i}, \ldots, x_{P}\right)-M_{j}\left(x_{1}, \ldots, x_{P}\right)}{\Delta x_{i}},
$$

where $\Delta x_{i}$ is a small increment, which we take to be some fraction of $x_{i}$, in other words, $\Delta x_{i}=f_{i} x_{i}$ where $f_{i} \in(0,1)$. However, to do comparison between different parameters and different variables which have different units and are on different orders of magnitude, we nondimensionalize $S_{i j}$ and convert it to a percentage

$$
\hat{S}_{i j} \approx\left|\frac{x_{i}}{M_{j}\left(x_{1}, \ldots, x_{P}\right)} S_{i j}\right| \times 100 .
$$

From here on out, we drop the hat notation and whenever we are referring to the sensitivity index $S_{i j}$, we are referring to (28).

When analyzing model (1)-(2), we assume that parameters $V_{a}^{0}, V_{v}^{0}, V_{d}^{0}$, and $V_{s}^{0}$ are fixed. Thus, because $C_{s}=-\frac{V_{s}^{0}}{P_{a}(0)}$, we also consider $C_{s}$ to be fixed. The parameters that we vary are $R_{a}, R_{c}, R_{p}, C_{a}, C_{v}$, and $C_{d}$.

\subsection{Algorithm}

The first step of the LH-OAT method is the LH portion. First, choose the number of Latin hypercube points that are to be used. Let $N$ be the number of Latin hypercube points and $k$ be the associated index. We use the MatLaB function lhsdesign with the default "maxmin" criterion (which maximizes minimum distance between points) to obtain LH points whose range is $(0,1)$. Because we use the following ranges for the parameters

$$
\begin{gathered}
R_{a} \in(0,2), \quad R_{c} \in(0,30), \quad R_{p} \in(0,5), \\
C_{a}, C_{v}, C_{d} \in(0,0.1),
\end{gathered}
$$

then the MATLAB-produced LH points are scaled to the desired ranges as specified.

The second step of the LH-OAT method is the OAT portion. Let $P$ be the number of parameters and $i$ be the associated index. Let $V$ be the number of model outputs (variables) and $j$ be the associated index. We examined two different forms of the sensitivity index $S_{i j}^{k}$. The first form is from [7], which is discretized as

$$
S_{i j}^{k}=\left|\frac{1}{f_{i}} \frac{M_{j}^{k}\left(x_{1}, \ldots, x_{i}\left(1+f_{i}\right), \ldots, x_{P}\right)-M_{j}^{k}\left(x_{1}, \ldots, x_{P}\right)}{\left[M_{j}^{k}\left(x_{1}, \ldots, x_{i}\left(1+f_{i}\right), \ldots, x_{P}\right)+M_{j}^{k}\left(x_{1}, \ldots, x_{P}\right)\right] / 2}\right| \times 100 .
$$




\begin{tabular}{|c|c|c|c|c|c|c|}
\hline & $R_{a}$ & $R_{c}$ & $R_{p}$ & $C_{a}$ & $C_{v}$ & $C_{d}$ \\
\hline $\bar{P}_{a}$ & 0.007 & 0.262 & -0.120 & -0.728 & -0.138 & -0.106 \\
\hline $\bar{Q}_{c}$ & -0.047 & -0.533 & -0.274 & -0.283 & -0.085 & -0.119 \\
\hline
\end{tabular}

Table 4: Correlation between parameters and variables

The other form is from $[4,5]$, which is discretized as

$$
S_{i j}^{k}=\left|\frac{1}{f_{i}} \frac{M_{j}^{k}\left(x_{1}, \ldots, x_{i}\left(1+f_{i}\right), \ldots, x_{P}\right)-M_{j}^{k}\left(x_{1}, \ldots, x_{P}\right)}{M_{j}^{k}\left(x_{1}, \ldots, x_{P}\right)}\right| \times 100 .
$$

For the resistance parameters $R_{j}$ we set the fractions $f_{i}=0.1$, and for the compliance parameters $C_{j}$ we set the fractions $f_{i}=0.001$.

The means and standard deviations for parameter $i$ and model output (variable) $j$ over all of the LH points are calculated as

$$
\begin{aligned}
\text { mean: } \quad \bar{S}_{i j} & =\frac{1}{N} \sum_{k=1}^{N} S_{i j}^{k}, \\
\text { standard deviation: } \quad \sigma_{i j} & =\sqrt{\frac{1}{N} \sum_{k=1}^{N}\left(S_{i j}^{k}-\bar{S}_{i j}\right)^{2}} .
\end{aligned}
$$

\subsection{Results}

We first plot the results of the $N=1000$ base simulations to see the effect of different parameters on model outputs in Figure 7. The two model outputs (variables) of interest, the average arterial pressure $\left(\bar{P}_{a}\right)$ and the average cardiac output $\left(\bar{Q}_{c}\right)$, are plotted against the parameter values $R_{a}, R_{c}, R_{p}, C_{a}, C_{v}$, and $C_{d}$.

The correlation between the parameters and the variables are given in Table 4, using the sensitivity form (30). We see that $C_{a}$ and $\bar{P}_{a}$ are strongly negatively correlated, $R_{c}$ and $\bar{Q}_{c}$ are moderately negatively correlated, and all other parameters and variables are weakly correlated.

We display the mean \pm standard deviation of the sensitivity indices $S_{i j}$ in Figure 8 using the sensitivity form (30). The largest mean corresponds with the strongest influence in the model output while the lowest mean corresponds with the weakest influence in the model output. Thus, we find that the ranking of strongest to weakest influence for $\bar{P}_{a}$ is

$$
C_{a}, \quad C_{v}, \quad R_{a}, \quad R_{c}, \quad R_{p}, \quad C_{d},
$$

while the ranking of strongest to weakest influence for $\bar{Q}_{c}$ is

$$
R_{a}, \quad R_{p}, \quad C_{a}, \quad C_{v}, \quad C_{d}, \quad R_{c}
$$

\section{Extended spatially homogeneous ODE model}

We extended the model (1)-(2) to account for differences in flow and pressure between the upper and lower parts of the body and to enable us to study the influence of the synthetic extracardiac conduit, which connects the inferior vena cava to the pulmonary arteries. In Figure 9, we show the resulting analogous electrical circuit diagram. We know that vessel resistances and compliances can change with age and vessel growth, but that these parameters remains constant for the extracardiac conduit. One of the objectives of this extended model was to determine if relative differences in resistances and compliances between the inferior vena cava and the extracardiac conduit led to differences in flows and pressures. 

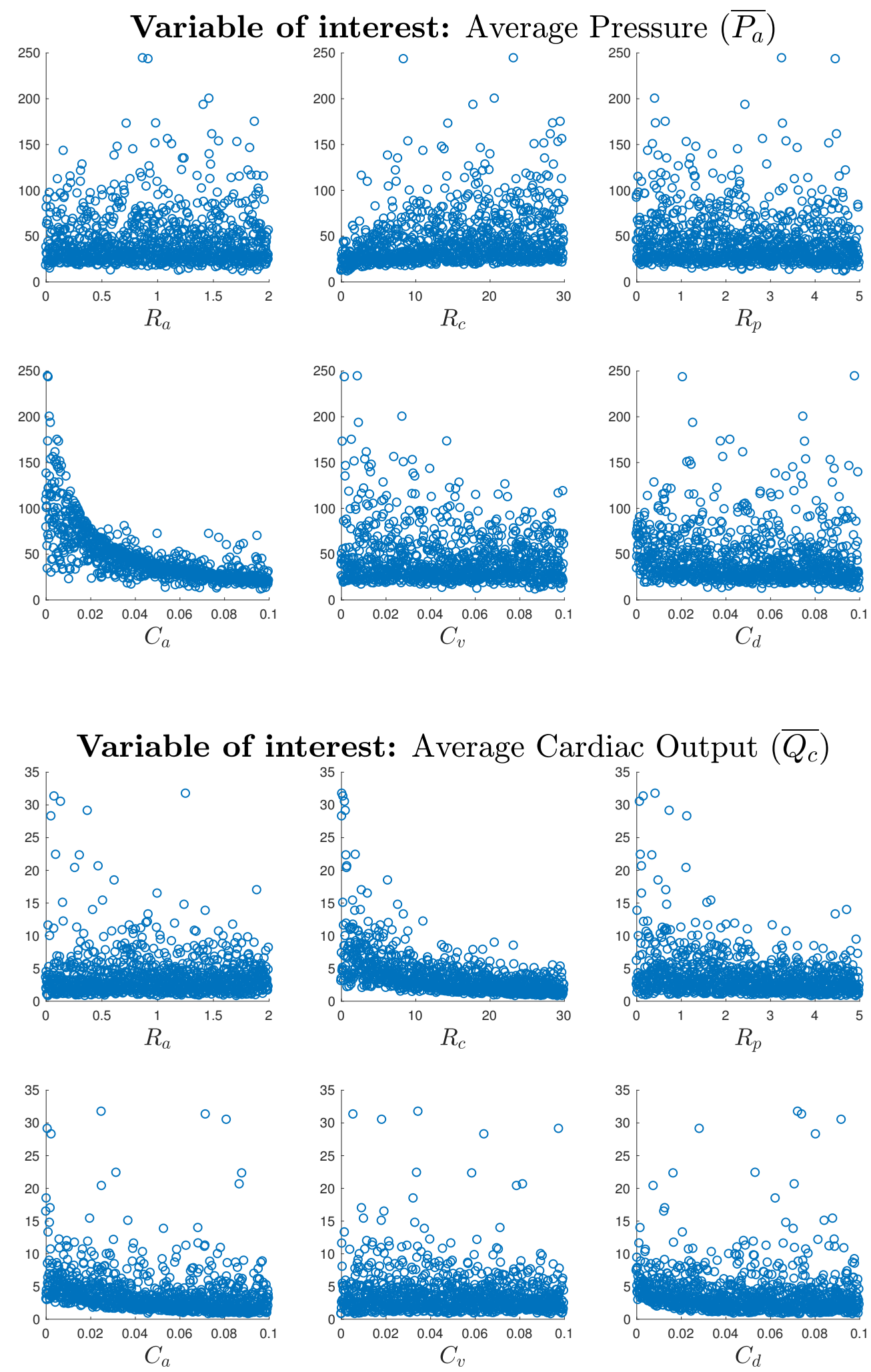

Figure 7: Scatter plot showing the effect of parameters on a variable of interest using the LH-OAT method. The two model outputs (variables) of interest are plotted against the parameter values using $N=1000$ base simulations. 


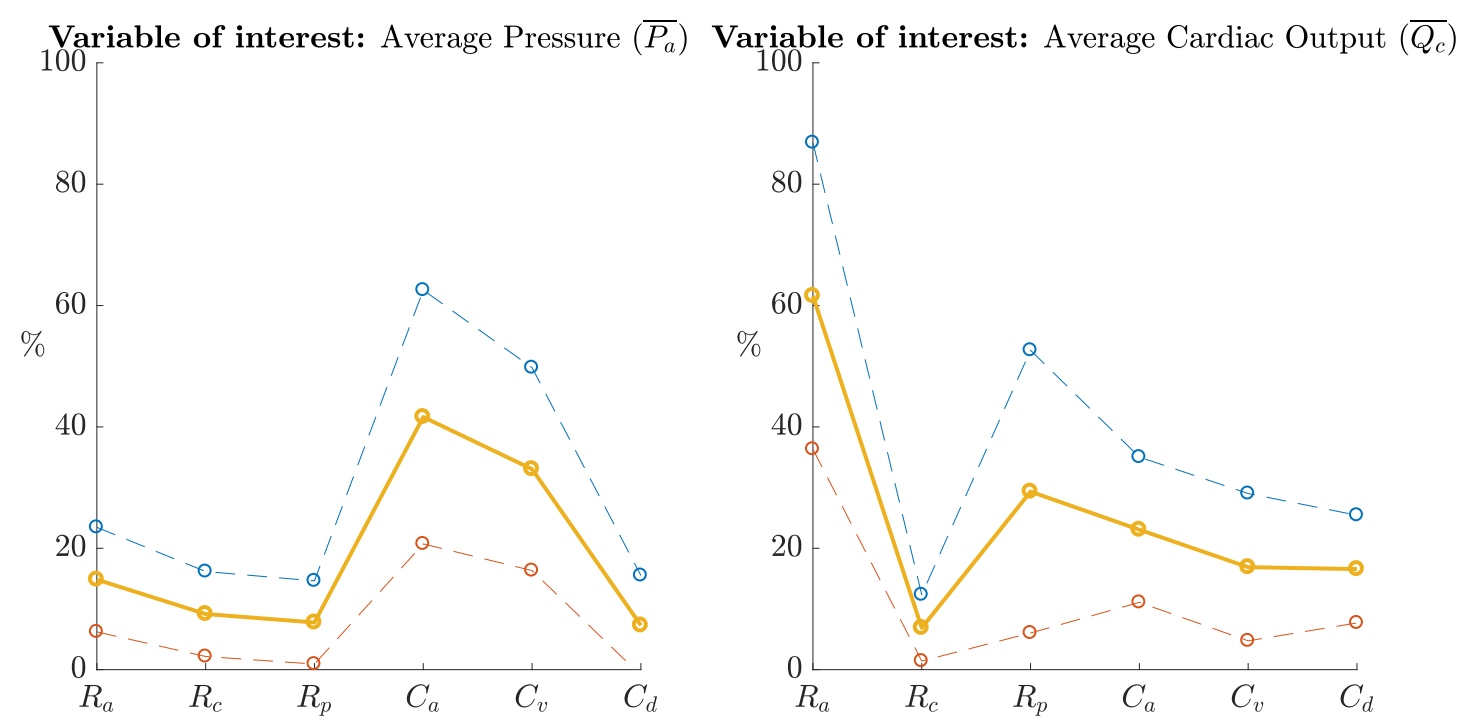

Figure 8: Mean (solid thick line) \pm standard deviation (dashed line) of the sensitivity indices $S_{i j}$ using the sensitivity form (30).

For this model, the governing equations for the flow rates are

$$
\begin{aligned}
& Q_{A 0}=\frac{P_{A 01}-P_{A 02}}{R_{A 0}}, \quad Q_{a, l b}=\frac{P_{A 02}-P_{a, l b}}{R_{a, l b}}, \quad Q_{c, l b}=\frac{P_{a, l b}-P_{\mathrm{IVC}}}{R_{c, l b}}, \\
& Q_{\mathrm{IVC}}=\frac{P_{\mathrm{IVC}}-P_{\mathrm{ECC}}}{R_{\mathrm{IVC}}}, \quad Q_{\mathrm{ECC}}=\frac{P_{\mathrm{ECC}}-P_{L}}{R_{\mathrm{ECC}}}, \quad Q_{p}=\frac{P_{L}-P_{p v}}{R_{p}}, \\
& Q_{a, u b}=\frac{P_{A 02}-P_{a, u b}}{R_{a, u b}}, \quad Q_{c, u b}=\frac{P_{a, u b}-P_{\mathrm{SVC}}}{R_{c, u b}}, \quad Q_{\mathrm{SVC}}=\frac{P_{\mathrm{SVC}}-P_{L}}{R_{\mathrm{SVC}}} .
\end{aligned}
$$

where $j=A 0$ corresponds to the aorta, $j=A 01$ corresponds to right after the heart but right before the aorta, $j=A 02$ corresponds to right after the aorta but right before the arterial vessels, $j=a, l b$ corresponds to arterial vessels in the lower body, $j=a, u b$ corresponds to arterial vessels in the upper body, $j=c, l b$ corresponds to capillaries in the lower body, $j=c, u b$ corresponds to capillaries in the upper body, $j=\mathrm{IVC}$ corresponds to the inferior vena cava, $j=\mathrm{SVC}$ corresponds to the superior vena cava, $j=$ ECC corresponds to extracardiac conduit, $j=L$ correspond to right after the superior vena cava and extracardiac conduit but right before the pulmonary vein, $j=p$ and $j=p v$ corresponds to the pulmonary vein, $j=d$ corresponds to the heart during diastole, and $j=s$ corresponds to the heart during systole.

The governing equations for the volumes are

$$
\begin{array}{rlrl}
V_{A 0}=V_{A 0}^{0}+C_{A 0} P_{A 02}, & V_{a, l b} & =V_{a, l b}^{0}+C_{a, l b} P_{a, l b}, & V_{\mathrm{IVC}}=V_{\mathrm{IVC}}^{0}+C_{\mathrm{IVC}} P_{\mathrm{IVC}}, \\
V_{\mathrm{ECC}}=V_{\mathrm{ECC}}^{0}+C_{\mathrm{ECC}} P_{\mathrm{ECC}}, & V_{a, u b} & =V_{a, u b}^{0}+C_{a, u b} P_{a, u b}, & V_{\mathrm{SVC}}=V_{\mathrm{SVC}}^{0}+C_{\mathrm{SVC}} P_{\mathrm{SVC}}, \\
V_{h} & = \begin{cases}V_{h s}^{0}+C_{s} P_{A 01} & \text { systole, } \\
V_{h d}^{0}+C_{d} P_{p v} & \text { diastole. }\end{cases} &
\end{array}
$$

and the governing equations for the pressures are 


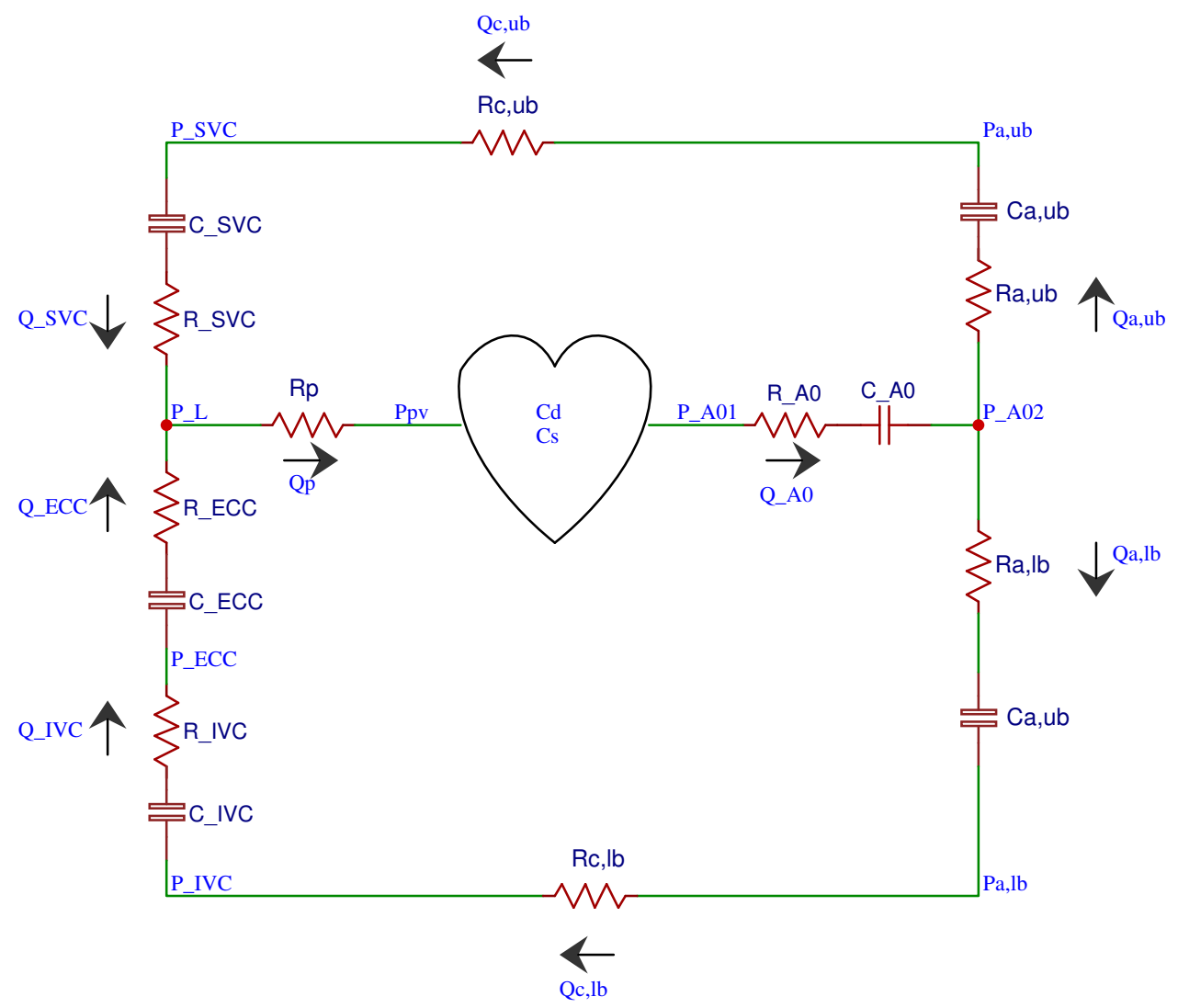

Figure 9: Diagram of the analogous electrical circuit model of the extended ODE model. $R_{j}$ represents the resistances of the blood vessels, $C_{j}$ represents the compliances, $P_{j}$ represents the blood pressures, and $Q_{j}$ represents the blood flows.

Diastole (inflow) $0<t<t_{1}$

$$
\begin{aligned}
C_{A 0} \frac{\mathrm{d} P_{A 02}}{\mathrm{~d} t} & =Q_{A 0}-\left(Q_{a, l b}+Q_{a, u b}\right), \\
C_{a, l b} \frac{\mathrm{d} P_{a, l b}}{\mathrm{~d} t} & =Q_{a, l b}-Q_{c, l b}, \\
C_{\mathrm{IVC}} \frac{\mathrm{d} P_{\mathrm{IVC}}}{\mathrm{d} t} & =Q_{c, l b}-Q_{\mathrm{IVC}}, \\
C_{\mathrm{ECC}} \frac{\mathrm{d} P_{\mathrm{ECC}}}{\mathrm{d} t} & =Q_{\mathrm{IVC}}-Q_{\mathrm{ECC}}, \\
C_{a, u b} \frac{\mathrm{d} P_{a, u b}}{\mathrm{~d} t} & =Q_{a, u b}-Q_{c, u b}, \\
C_{\mathrm{SVC}} \frac{\mathrm{d} P_{\mathrm{SVC}}}{\mathrm{d} t} & =Q_{c, u b}-Q_{\mathrm{SVC}}, \\
C_{d} \frac{\mathrm{d} P_{p v}}{\mathrm{~d} t} & =Q_{p},
\end{aligned}
$$

Systole (outflow) $t_{1}<t<t_{2}$

$$
\begin{aligned}
C_{A 0} \frac{\mathrm{d} P_{A 02}}{\mathrm{~d} t} & =Q_{A 0}-\left(Q_{a, l b}+Q_{a, u b}\right), \\
C_{a, l b} \frac{\mathrm{d} P_{a, l b}}{\mathrm{~d} t} & =Q_{a, l b}-Q_{c, l b}, \\
C_{\mathrm{IVC}} \frac{\mathrm{d} P_{\mathrm{IVC}}}{\mathrm{d} t} & =Q_{c, l b}-Q_{\mathrm{IVC}}, \\
C_{\mathrm{ECC}} \frac{\mathrm{d} P_{\mathrm{ECC}}}{\mathrm{d} t} & =Q_{\mathrm{IVC}}-Q_{\mathrm{ECC}}, \\
C_{a, u b} \frac{\mathrm{d} P_{a, u b}}{\mathrm{~d} t} & =Q_{a, u b}-Q_{c, u b}, \\
C_{\mathrm{SVC}} \frac{\mathrm{d} P_{\mathrm{SVC}}}{\mathrm{d} t} & =Q_{c, u b}-Q_{\mathrm{SVC}}, \\
C_{s} \frac{\mathrm{d} P_{A 01}}{\mathrm{~d} t} & =-Q_{A 0} .
\end{aligned}
$$


The system conditions are

$$
\begin{aligned}
& V_{T}=V_{A 0}+V_{a, l b}+V_{\mathrm{IVC}}+V_{\mathrm{ECC}}+V_{a, u b}+V_{\mathrm{SVC}}+V_{h} \\
& Q_{A 0}=0 \quad \Longrightarrow \quad P_{A 01}=P_{A 02} \text {, during diastole, } \\
& Q_{p}=0 \quad \Longrightarrow \quad P_{L}=P_{p v}, \quad \text { during systole, } \\
& Q_{\mathrm{ECC}}+Q_{\mathrm{SVC}}=Q_{p} \Longrightarrow \\
& P_{L}= \begin{cases}\frac{R_{\mathrm{ECC}} R_{p} P_{\mathrm{SVC}}+R_{\mathrm{ECC}} R_{\mathrm{SVC}} P_{p v}+R_{p} R_{\mathrm{IVC}} P_{\mathrm{ECC}}}{R_{p} R_{\mathrm{SVC}}+R_{\mathrm{ECC}} R_{p}+R_{\mathrm{ECC}} R_{\mathrm{SVC}}}, & \text { during diastole } \\
\frac{R_{\mathrm{ECC}} P_{\mathrm{SVC}}+R_{\mathrm{SVC}} P_{\mathrm{ECC}}}{R_{\mathrm{ECC}}+R_{\mathrm{SVC}}}, & \text { during systole }\end{cases}
\end{aligned}
$$

These equations can then be simplified to

Diastole (inflow) $0<t<t_{1}$

$$
\begin{aligned}
C_{A 0} \frac{\mathrm{d} P_{A 02}}{\mathrm{~d} t}=-\frac{P_{A 02}-P_{a, l b}}{R_{a, l b}}-\frac{P_{A 02}-P_{a, u b}}{R_{a, u b}}, & C_{A 0} \frac{\mathrm{d} P_{A 02}}{\mathrm{~d} t}=\frac{P_{A 01}-P_{A 02}}{R_{A 0}}-\frac{P_{A 02}-P_{a, l b}}{R_{a, l b}}-\frac{P_{A 02}-P_{a, u b}}{R_{a, u b}}, \\
C_{a, l b} \frac{\mathrm{d} P_{a, l b}}{\mathrm{~d} t}=\frac{P_{A 02}-P_{a, l b}}{R_{a, l b}}-\frac{P_{a, l b}-P_{\mathrm{IVC}}}{R_{c, l b}}, & C_{a, l b} \frac{\mathrm{d} P_{a, l b}}{\mathrm{~d} t}=\frac{P_{A 02}-P_{a, l b}}{R_{a, l b}}-\frac{P_{a, l b}-P_{\mathrm{IVC}}}{R_{c, l b}}, \\
C_{\mathrm{IVC}} \frac{\mathrm{d} P_{\mathrm{IVC}}}{\mathrm{d} t}=\frac{P_{a, l b}-P_{\mathrm{IVC}}}{R_{c, l b}}-\frac{P_{\mathrm{IVC}}-P_{\mathrm{ECC}}}{R_{\mathrm{IVC}}}, & C_{\mathrm{IVC}} \frac{\mathrm{d} P_{\mathrm{IVC}}}{\mathrm{d} t}=\frac{P_{a, l b}-P_{\mathrm{IVC}}}{R_{c, l b}}-\frac{P_{\mathrm{IVC}}-P_{\mathrm{ECC}}}{R_{\mathrm{IVC}}}, \\
C_{\mathrm{ECC}} \frac{\mathrm{d} P_{\mathrm{ECC}}}{\mathrm{d} t}=\frac{P_{\mathrm{IVC}}-P_{\mathrm{ECC}}}{R_{\mathrm{IVC}}}-\frac{P_{\mathrm{ECC}}-P_{L}}{R_{\mathrm{ECC}}}, & C_{\mathrm{ECC}} \frac{\mathrm{d} P_{\mathrm{ECC}}}{\mathrm{d} t}=\frac{P_{\mathrm{IVC}}-P_{\mathrm{ECC}}}{R_{\mathrm{IVC}}}-\frac{P_{\mathrm{ECC}}-P_{L}}{R_{\mathrm{ECC}}}, \\
C_{a, u b} \frac{\mathrm{d} P_{a, u b}}{\mathrm{~d} t}=\frac{P_{A 02}-P_{a, u b}}{R_{a, u b}}-\frac{P_{a, u b}-P_{\mathrm{SVC}}}{R_{c, u b}}, & C_{a, u b} \frac{\mathrm{d} P_{a, u b}}{\mathrm{~d} t}=\frac{P_{A 02}-P_{a, u b}}{R_{a, u b}}-\frac{P_{a, u b}-P_{\mathrm{SVC}}}{R_{c, u b}}, \\
C_{\mathrm{SVC}} \frac{\mathrm{d} P_{\mathrm{SVC}}}{\mathrm{d} t}=\frac{P_{a, u b}-P_{\mathrm{SVC}}}{R_{c, u b}}-\frac{P_{\mathrm{SVC}}-P_{L}}{R_{\mathrm{SVC}}}, & C_{\mathrm{SVC}} \frac{\mathrm{d} P_{\mathrm{SVC}}}{\mathrm{d} t}=\frac{P_{a, u b}-P_{\mathrm{SVC}}}{R_{c, u b}}-\frac{P_{\mathrm{SVC}}-P_{L}}{R_{\mathrm{SVC}}},
\end{aligned}
$$

Systole (outflow) $t_{1}<t<t_{2}$

where during diastole

$$
\begin{aligned}
P_{p v}= & \frac{1}{C_{d}}\left(V_{T}-V_{d}^{0}-C_{A 0} P_{A 02}-C_{a, l b} P_{a, l b}-C_{\mathrm{IVC}} P_{\mathrm{IVC}}\right. \\
P_{A 02}= & \left.P_{A 01}, \quad-C_{\mathrm{ECC}} P_{\mathrm{ECC}}-C_{a, u b} P_{a, u b}-C_{\mathrm{SVC}} P_{\mathrm{SVC}}\right), \\
V_{d}^{0}= & V_{A 0}^{0}+V_{a, l b}^{0}+V_{\mathrm{IVC}}^{0}+V_{\mathrm{ECC}}^{0}+V_{a, u b}^{0}+V_{\mathrm{SVC}}^{0}+V_{h d}^{0},
\end{aligned}
$$

and during systole

$$
\begin{aligned}
P_{A 01}= & \frac{1}{C_{s}}\left(V_{T}-V_{s}^{0}-C_{A 0} P_{A 02}-C_{a, l b} P_{a, l b}-C_{\mathrm{IVC}} P_{\mathrm{IVC}}\right. \\
& \left.\quad-C_{\mathrm{ECC}} P_{\mathrm{ECC}}-C_{a, u b} P_{a, u b}-C_{\mathrm{SVC}} P_{\mathrm{SVC}}\right), \\
P_{L}= & P_{p v}, \quad \\
V_{s}^{0} & =V_{A 0}^{0}+V_{a, l b}^{0}+V_{\mathrm{IVC}}^{0}+V_{\mathrm{ECC}}^{0}+V_{a, u b}^{0}+V_{\mathrm{SVC}}^{0}+V_{h s}^{0},
\end{aligned}
$$

Table 5 provides the typical parameter values used in this extended ODE model. 


\begin{tabular}{|c|c|c|c|}
\hline Parameters & Typical value & Units & Description \\
\hline$V_{A o}^{0}$ & 0.4 & $\mathrm{~L}$ & Basal aortic volume \\
\hline$V_{a, l b}^{0}$ & 0.3 & $\mathrm{~L}$ & Basal lower body arterial volume \\
\hline$V_{a, u b}^{0, u}$ & 0.3 & $\mathrm{~L}$ & Basal upper body arterial volume \\
\hline$V_{\mathrm{SVC}}^{0}$ & 0.9 & $\mathrm{~L}$ & Basal superior vena cava volume \\
\hline$V_{\mathrm{IVC}}^{0}$ & 0.8 & $\mathrm{~L}$ & Basal inferior vena cava volume \\
\hline$V_{\mathrm{ECC}}^{0}$ & 0.8 & $\mathrm{~L}$ & Basal extracardiac conduit volume \\
\hline$V_{d}^{0}$ & 0 & $\mathrm{~L}$ & Basal heart volume in diastolic phase \\
\hline$V_{s}^{0}$ & -0.5 & $\mathrm{~L}$ & Basal heart volume in systolic phase \\
\hline$V_{T}$ & 5.0 & $\mathrm{~L}$ & Total volume of the vessel system \\
\hline$C_{A o}$ & 0.0005 & $\operatorname{mmHg} \min \mathrm{L}^{-1}$ & Aortic compliance \\
\hline$C_{a, l b}$ & 0.0005 & $\operatorname{mmHg} \min \mathrm{L}^{-1}$ & Lower body arterial compliance \\
\hline$C_{a, u b}$ & 0.0005 & $\operatorname{mmHg} \min \mathrm{L}^{-1}$ & Upper body arterial compliance \\
\hline$C_{\mathrm{SVC}}$ & 0.18 & $\mathrm{mmHg} \min \mathrm{L}^{-1}$ & Superior vena cava compliance \\
\hline$C_{\mathrm{IVC}}$ & 0.18 & $\mathrm{mmHg} \min \mathrm{L}^{-1}$ & Inferior vena cava compliance \\
\hline$C_{\mathrm{ECC}}$ & 0.18 & $\mathrm{mmHg} \min \mathrm{L}^{-1}$ & Extracardiac conduit compliance \\
\hline$C_{d}$ & 0.01 & $\mathrm{~L} \mathrm{mmHg}^{-1}$ & Heart compliance during diastole \\
\hline$C_{s}$ & 0.005 & $\mathrm{~L} \mathrm{mmHg}^{-1}$ & Heart compliance during systole \\
\hline$R_{A o}$ & 0.5 & $\operatorname{mmHg} \min \mathrm{L}^{-1}$ & Aortic resistance \\
\hline$R_{a, l b}$ & 1.0 & $\mathrm{mmHg} \min \mathrm{L}^{-1}$ & Lower body arterial resistance \\
\hline$R_{a, u b}$ & 2.0 & $\operatorname{mmHg} \min \mathrm{L}^{-1}$ & Upper body arterial resistance \\
\hline$R_{c, l b}$ & 34 & $m m H g \min L^{-1}$ & Lower body capillary resistance \\
\hline$R_{c, u b}$ & 34 & $\operatorname{mmHg} \min \mathrm{L}^{-1}$ & Upper body capillary resistance \\
\hline$R_{\mathrm{SVC}}$ & 0.2 & $\operatorname{mmHg} \min \mathrm{L}^{-1}$ & Superior vena cava resistance \\
\hline$R_{\mathrm{IVC}}$ & 0.2 & $m m H g \min \mathrm{L}^{-1}$ & Inferior vena cava resistance \\
\hline$R_{\mathrm{ECC}}$ & 0.002 & $\mathrm{mmHg} \min \mathrm{L}^{-1}$ & Extracardiac conduit resistance \\
\hline$R_{p}$ & 2.9 & $m m H g \min \mathrm{L}^{-1}$ & Pulmonary resistance \\
\hline
\end{tabular}

Table 5: Table of the typical parameter values of the extended ODE model.

\subsection{Results}

In order to solve the extended model we implemented a numerical solver very similar to the one described in Section 5, as the method to solve the two ODEs of the small model can be easily extended to solve the system of six ODEs presented in Section 7.

Typical results for the extended ODE model are shown in Figure 10. Overall, these results are consistent with those found for the the original ODE model, as shown in Figure 4. There were several interesting findings that warrant further investigation. Specifically, it was observed that $P_{\mathrm{IVC}} \approx P_{\mathrm{SVC}}$, but $P_{\mathrm{IVC}} \neq P_{\mathrm{ECC}}$, which is unexpected given that the extracardiac conduit lies between the IVC and the SVC. Additionally, it was surprising to observe that the ratio of $Q_{a, u b} / Q_{a, l b}$ was $>1$ or $<1$ during different parts of the cardiac cycle. It is known on average that approximately two-thirds of the blood flow travels to the lower body and one third to the upper body (i.e. $Q_{a, u b} / Q_{a, l b} \approx 0.5$ ), so the portions where $Q_{a, u b} / Q_{a, l b}>1$ were unexpected and warrant further investigation. 

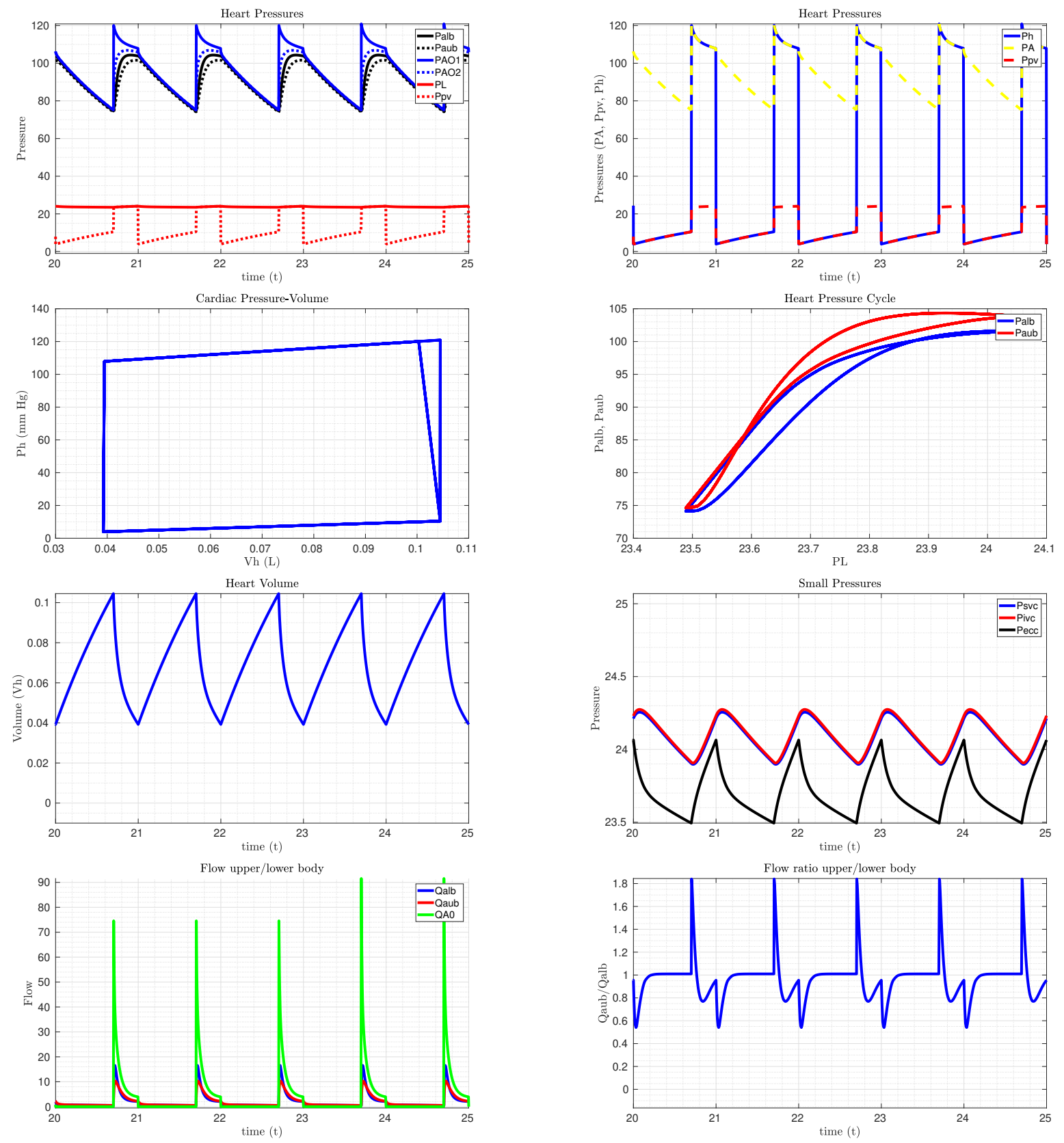

Figure 10: Typical output for a single run of the extended ODE model. Parameter values are as stated in Table 5. 


\section{Spatially inhomogeneous PDE model}

We now consider the spatially inhomogeneous PDE model from [1] for the pressure in a Fontan blood flow circulation. This model takes into account the spatial variations of the model parameters, such as the compliances and resistances, and is defined as

$$
\begin{gathered}
C \frac{\partial P}{\partial t}=\frac{\partial}{\partial x}\left(q(x, P) \frac{\partial P}{\partial x}\right) \\
q(x, P)=N\left(A_{0}+C P\right)^{2}
\end{gathered}
$$

where $P(x, t)$ is the pressure, $C(x)$ is the compliance, $A_{0}(x)$ is the cross-sectional area of a blood vessel, and $N(x)$ is the total number of parallel vessels with the same cross-sectional area.

The model (38) is solved subject to boundary and initial conditions, as summarized below. The initial condition is

$$
P(x, 0)=P_{0}(x) .
$$

During the systolic regime, $0<t<t_{1}$, the boundary conditions are

$$
\begin{aligned}
q(0, P) \frac{\partial P}{\partial x}(0, t) & =C_{l s} \frac{\partial P}{\partial t}(0, t), \\
\frac{\partial P}{\partial x}(1, t) & =0 .
\end{aligned}
$$

During the diastole regime, $t_{1}<t<t_{2}$, the boundary conditions are

$$
\begin{gathered}
\frac{\partial P}{\partial x}(0, t)=0 \\
q(1, P) \frac{\partial P}{\partial x}(1, t)=C_{l d} \frac{\partial P}{\partial t}(1, t) .
\end{gathered}
$$

The problem is periodic in time, i.e., the systolic and diastolic regimes are repeated.

\subsection{Non-dimensionalzation}

We non-dimensionalized the model (38)-(41) to reduce the number of parameters and to enable us to estimate the importance of the various terms using the scalings

$$
x=L x^{*}, \quad t=T t^{*}, \quad P=P_{0} P^{*}, \quad C=C_{0} C^{*}, \quad N=N_{\max } N^{*}, \quad A_{0}=A_{\max } A_{0}^{*} .
$$

Dropping the asterisks, we obtain the dimensionless PDE

$$
\begin{aligned}
& C \frac{\partial P}{\partial t}=\frac{\partial}{\partial x}\left(q(x, P) \frac{\partial P}{\partial x}\right), \\
& q(x, P)=N\left(A_{0}+\alpha C P\right)^{2}
\end{aligned}
$$

where $\alpha=C_{0} P_{0} / A_{\max }$ and timescale $T=\left(8 \pi \mu c_{0} L^{2}\right) /\left(N_{\max } A_{\max }^{2}\right)$. During the systolic regime, $0<t<$ $T^{-1} t_{1}$, the boundary conditions are

$$
\begin{aligned}
q(0, P) \frac{\partial P}{\partial x}(0, t) & =\bar{C}_{l s} \frac{\partial P}{\partial t}(0, t), \\
\frac{\partial P}{\partial x}(1, t) & =0
\end{aligned}
$$




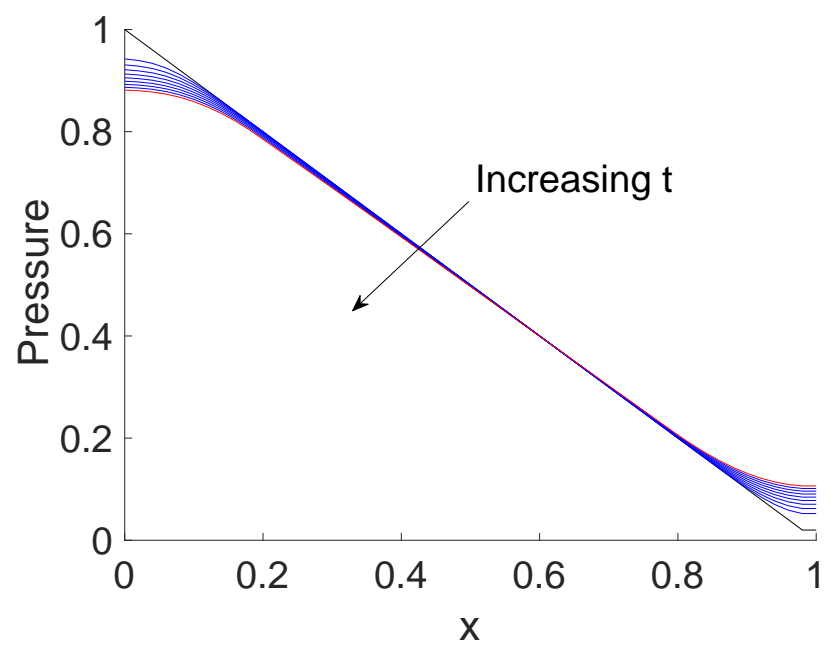

Figure 11: Pressure drop as a function of space under Fontan conditions.

where $\bar{C}_{l s}=C_{l s} / C_{0} L$. During the diastole regime, $T^{-1} t_{1}<t<T^{-1} t_{2}$, the boundary conditions are

$$
\begin{gathered}
\frac{\partial P}{\partial x}(0, t)=0 \\
q(1, P) \frac{\partial P}{\partial x}(1, t)=\bar{C}_{l d} \frac{\partial P}{\partial t}(1, t),
\end{gathered}
$$

where $\bar{C}_{l d}=C_{l d} / C_{0} L$.

\subsection{Results}

Simulations can be done by sequentially solving the PDE using a finite differences numerical method with fixed time step, switching periodically between different boundary conditions for systole and diastole. Figure 11 shows the pressure drop as a function of distance from the left-side of the heart, $x=0$ to the right side of the heart, $x=1$, at various times throughout the simulation. The good qualitative agreement with Figure 5 in [1] provides validation of our results.

\section{Summary and future work}

We have implemented and analyzed three lumped parameter models of the Fontan circulation: the original spatially homogeneous ODE model, the extended homogeneous ODE model, and the spatially inhomogeneous PDE model. We started by studying the ODE model presented in [1], both analytically and numerically. For the analytical solutions, even though exact solutions can be found, we looked for asymptotic approximations in the limit of large venous compliance, as they provide simpler and more insightful expressions for the solutions. The numerical solver was implemented using a variable time-stepping method in MatLAB. We found good agreement between the asymptotic solutions and the numerical simulations. We also considered the Poincaré maps for the pressure at the beginning of diastole and systole, which provide the pressure at the same point but one heartbeat later. These are useful to determine the convergence to a limit cycle and to construct analytical solutions over several heartbeats. From the Poincaré maps we conclude that the system always converges very quickly to a limit cycle. Parameter sensitivity analysis was done using the LH-OAT method and with a parameter sweep to find regions of "healthy" and "failing" Fontan circulation to identify 
parameters that have a significant effect on the model output. The extended ODE model was developed to separate the blood flow to the upper and lower parts of the body and study the impact of changing model parameters in the presence of the synthetic extracardiac conduit. Preliminary results have identified some surprising findings as discussed in Section 7.1 that warrant further investigation. Results from the PDE model show good qualitative agreement with previous findings and suggest further research to develop this model more completely.

In terms of future work, there are a number of possible directions for this project. Since the initial work summarized in this report, the problem presenter and collaborators have developed a modified version of the original ODE model, which is a reduction of a two ventricle model. This new model exists in three versions, two ventricle, healthy Fontan, and failing Fontan. One potential future direction is to apply the techniques developed in this work to these new models. A second possible future direction is to develop a new extended ODE model and apply the techniques developed in this work to that model. Lastly, further development of the PDE model could be undertaken to determine the impact of spatial variation of model parameters on the ability of the model to capture patient-specific physiological behavior and improve our understanding of Fontan failure.

\section{References}

[1] J. P. Keener, M. Chugunova, R. M. Taranets, and M. G. Doyle. Use of mathematical modeling to study pressure regimes in normal and Fontan blood flow circulations. arXiv:1806.02416, 2018.

[2] James Keener and James Sneyd. Mathematical Physiology: II: Systems Physiology. Springer, New York, NY, 2nd edition, 2009.

[3] Ethan Kung, Giancarlo Pennati, Francesco Migliavacca, Tain-Yen Hsia, Richard Figliola, Alison Marsden, and Alessandro Giardini. A simulation protocol for exercise physiology in Fontan patients using a closed loop lumped-parameter model. J. Biomech. Eng., 136(8):081007, 2014.

[4] Fuyou Liang, Hideaki Senzaki, Clara Kurishima, Koichi Sughimoto, Ryo Inuzuka, and Hao Liu. Hemodynamic performance of the Fontan circulation compared with a normal biventricular circulation: a computational model study. AJP-Heart Circ. Physiol., 307(7):H1056-H1072, 2014.

[5] Fuyou Liang, Koichi Sughimoto, Kozo Matsuo, Hao Liu, and Shu Takagi. Patient-specific assessment of cardiovascular function by combination of clinical data and computational model with applications to patients undergoing Fontan operation. Int. J. Numer. Meth. Biomed. Eng., 30(10):1000-1018, 2014.

[6] Amy L Throckmorton, James P Carr, Sharjeel A Tahir, Ryan Tate, Emily A Downs, Sonya S Bhavsar, Yi Wu, John D Grizzard, and William B Moskowitz. Mechanical cavopulmonary assistance of a patientspecific Fontan physiology: numerical simulations, lumped parameter modeling, and suction experiments. Artif. Organs, 35(11):1036-1047, 2011.

[7] A. van Griensven, T. Meixner, S. Grunwald, T. Bishop, M. Diluzio, and R. Srinivasan. A global sensitivity analysis tool for the parameters of multi-variable catchment models. J. Hydrol., 324(1):10-23, 2006. 